\title{
IP Eridani: A surprising long-period binary system hosting a He white dwarf ${ }^{\star}, \star \star$
}

\author{
T. Merle ${ }^{1}$, A. Jorissen ${ }^{1}$, T. Masseron ${ }^{1}$, S. Van Eck ${ }^{1}$, L. Siess ${ }^{1}$, and H. Van Winckel ${ }^{2}$ \\ ${ }^{1}$ Institut d'Astronomie et d'Astrophysique, Université Libre de Bruxelles, CP 226, boulevard du Triomphe, 1050 Brussels, Belgium \\ e-mail: tmerle@ulb.ac.be \\ 2 Instituut voor Sterrenkunde, Katholieke Universiteit Leuven, Celestijnenlaan 200D, 3200 Heverlee, Belgium
}

Received 13 March 2014 / Accepted 15 May 2014

\begin{abstract}
We determine the orbital elements for the K0 IV + white dwarf (WD) system IP Eri, which appears to have a surprisingly long period of $1071 \mathrm{~d}$ and a significant eccentricity of 0.25 . Previous spectroscopic analyses of the WD, based on a distance of $101 \mathrm{pc}$ inferred from its HIPPARCOS parallax, yielded a mass of only $0.43 M_{\odot}$, implying it to be a helium-core WD. The orbital properties of IP Eri are similar to those of the newly discovered long-period subdwarf B star (sdB) binaries, which involve stars with Heburning cores surrounded by extremely thin $\mathrm{H}$ envelopes, and are therefore close relatives to He WDs. We performed a spectroscopic analysis of high-resolution spectra from the HERMES/Mercator spectrograph and concluded that the atmospheric parameters of the $\mathrm{K} 0$ component are $T_{\mathrm{eff}}=4960 \mathrm{~K}, \log g=3.3,[\mathrm{Fe} / \mathrm{H}]=0.09$ and $\xi=1.5 \mathrm{~km} \mathrm{~s}^{-1}$. The detailed abundance analysis focuses on $\mathrm{C}, \mathrm{N}$, $\mathrm{O}$ abundances, carbon isotopic ratio, light ( $\mathrm{Na}, \mathrm{Mg}, \mathrm{Al}, \mathrm{Si}, \mathrm{Ca}, \mathrm{Ti})$ and s-process ( $\mathrm{Sr}, \mathrm{Y}, \mathrm{Zr}, \mathrm{Ba}, \mathrm{La}, \mathrm{Ce}, \mathrm{Nd})$ elements. We conclude that IP Eri abundances agree with those of normal field stars of the same metallicity. The long period and non-null eccentricity indicate that this system cannot be the end product of a common-envelope phase; it calls instead for another less catastrophic binary-evolution channel presented in detail in a companion paper.
\end{abstract}

Key words. stars: abundances - binaries: spectroscopic - stars: evolution - stars: individual: IP Eridani - subdwarfs - white dwarfs

\section{Introduction}

IP Eri is a very interesting system consisting of a $\mathrm{K} 0$ (sub)giant and a He white dwarf (WD) and it is tempting to relate this system to the family of subdwarf B ( $\mathrm{sdB}$ ) binaries where the hot component is a bare He-burning core surrounded by an extremely thin $\mathrm{H}$ envelope (Heber 2009). These systems owe their properties to envelope ejection, likely due to binary interaction, as they evolve along the red giant branch. Their evolution shares some similarities with that of IP Eri and such systems are important benchmarks for binary evolution.

Several long-period eccentric systems (with $P \sim 10^{3} \mathrm{~d}$ ) were recently discovered among sdB stars $\left(\varnothing_{\text {stensen } \& \text { Van Winckel }}\right.$ 2011, 2012; Vos et al. 2012, 2013; Deca et al. 2012; Barlow et al. 2012, 2013). Using new radial-velocity data collected with the HERMES/Mercator spectrograph (Raskin et al. 2011), we show in this paper (Sect. 3) that IP Eri adds to this new class of long-period eccentric systems.

Since the He-WD progenitor did not evolve along the asymptotic giant branch (AGB), it had no chance to produce s-process elements and to pollute its companion (the present K0 subgiant), so that the latter should not appear as a barium star (a family of $\mathrm{K}$ giants with enhanced abundances of s-process elements; Bidelman \& Keenan 1951). It is therefore of interest to perform a chemical analysis of the K0 subgiant in the IP Eri system to confirm the absence of overabundances of s-process elements.

\footnotetext{
$\star$ Appendix A is available in electronic form at http://www . aanda.org

$\star \star$ HERMES spectra are only available at the CDS via anonymous ftp to cdsarc.u-strasbg. fr $(130.79 .128 .5)$ or via http://cdsarc.u-strasbg.fr/viz-bin/qcat?]/A+A/567/A30
}

This is the second objective of the present paper, which is organized as follows: Sect. 2 gives an overview of the properties of the IP Eri system. Sect. 3 presents the radial-velocity data and the ensuing orbit. After deriving the atmospheric parameters of IP Eri in Sect. 4, the abundance analysis is presented in Sect. 5, with emphasis on s-process elements. These abundances are then compared with expectations for barium stars and for non-s-process-polluted stars, as derived from the abundance trends observed in large samples of field stars reflecting the chemical evolution of the Galaxy (Sect. 6). Section 7 briefly confronts our results with predictions from binary-evolution scenarios presented in detail in a companion paper (Siess et al. 2014). Section 8 summarizes our results.

\section{The IP Eri system}

IP Eri (HD 18131, HIP 13558, WD 0252-055, EUVE J0254-053) has attracted attention since its discovery as an extreme UV (EUV) source both by ROSAT (Pounds et al. 1993; Pye et al. 1995) and EUVE (Bowyer et al. 1994, 1996; Malina et al. 1994). In a subsequent analysis, Vennes et al. (1995) find that an older International Ultraviolet Explorer (IUE) spectrum already revealed that a WD dominates the IUE spectrum below $200 \mathrm{~nm}$ while a $\mathrm{K} 0$ spectrum prevails at longer wavelengths. Their model-atmosphere analysis of the EUV photometry reveals a hot, hydrogen-rich (DA) WD (with an effective temperature of about $30000 \mathrm{~K}$ ) that is the most likely source of the EUV emission. In the grid of models fitted to the WD spectrum by Burleigh et al. (1997), the model with a temperature of $29290 \mathrm{~K}$, a gravity of $\log g=7.5$ and a mass of $0.43 M_{\odot}$ locates the WD at a distance of $93 \mathrm{pc}$, consistent with the HIPPARCOS 


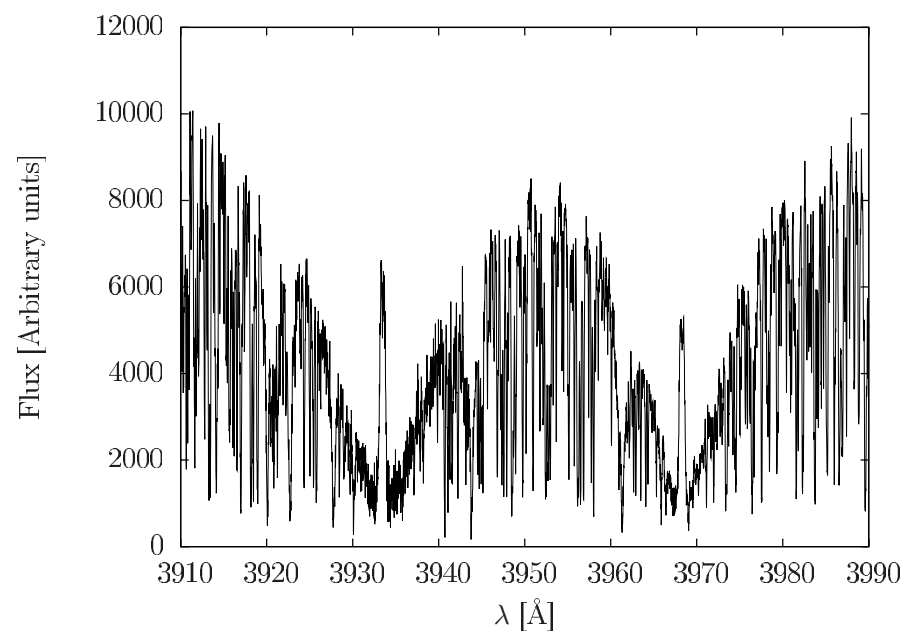

Fig. 1. The Ca II $\mathrm{H}$ and $\mathrm{K}$ lines, from the HERMES spectrum on HJD 2455066.748 (2009, August 23). The emission present in the cores is somewhat more intense than the one presented in Fig. 5 of Vennes et al. (1997).

parallax of the $\mathrm{K}$ star, as discussed below. The corresponding age for the WD is then 7 Myr (Burleigh et al. 1997). The WD parameters inferred by Burleigh et al. (1997) locate it among He WDs in the $\log g-\log T_{\text {eff }}$ diagram of Driebe et al. (1998). Its mass of $0.43 M_{\odot}$ is incompatible with a CO WD since the minimum CO core mass at the base of the AGB (just at the end of core He-burning) is $0.51 M_{\odot}$ (for a star of initial mass $0.9 M_{\odot}$; e.g., Eq. (66) of Hurley et al. 2000). An independent study by Vennes et al. (1998) concludes at a somewhat higher mass $\left(0.48-0.52 M_{\odot}\right)$ for the IP Eri WD, which is still, for the most part of this range, compatible with the He nature of the WD.

The revised HIPPARCos parallax of $9.82 \pm 0.94$ mas corresponds to a distance of $101 \pm 11 \mathrm{pc}$ and a distance modulus of $5.03 \pm 0.2$ (van Leeuwen 2007). With $V=7.32$ (Cutispoto et al. 1995), one gets an absolute magnitude of 2.29 for the $\mathrm{K}$ star, which indicates that it is a subgiant. The photometric data for the K0 subgiant are $V=7.32, U-B=0.74, B-V=$ $0.98, V-R \mathrm{c}=0.52, V-I \mathrm{c}=0.97$ (Cutispoto et al. 1995) and $J=5.709, H=5.263, K=5.090$ (2MASS; Cutri et al. 2003). The corresponding $V-K$ index of 2.23 implies an effective temperature of $4900 \mathrm{~K}$ (Bessell et al. 1998). Using a Bayesian method, Bailer-Jones (2011) obtains $\log T_{\text {eff }}=3.70 \pm 0.01$ (5012 K) for IP Eri.

Although neither the ASAS lightcurve (Pojmanski 1997) nor the monitoring performed by Cutispoto et al. (1999) reveals variability at the $0.1 \mathrm{mag}$ level, a more accurate monitoring by Strassmeier et al. (2000) has uncovered a 0.045 mag variability over $30 \mathrm{~d}$ in the Strömgren $y$ band, hence its classification as a BY Dra variable in the General Catalogue of Variable Stars (with name IP Eri). The star is slowly rotating ( $V \sin i<5 \mathrm{~km} \mathrm{~s}^{-1}$; Cutispoto et al. 1999). Should the $30 \mathrm{~d}$ photometric variability be due to rotation, a value of $\sim 1 \mathrm{~km} \mathrm{~s}^{-1}$ for the rotational velocity would result from the radius of $3.8 R_{\odot}$ derived in (Siess et al. 2014) for IP Eri. Nevertheless, this star exhibits moderate $\mathrm{Mg}$ II $\mathrm{h}$ and $\mathrm{k}$ emission in the IUE spectrum and Ca II H \& K emission (Fig. 5 of Vennes et al. 1997; Strassmeier et al. 2000). Figure 1 shows the emission cores in the Ca II lines, as seen on our HERMES/Mercator spectrum obtained on HJD 2455066.748 (2009, August 23). This emission core is somewhat more intense than the one presented by Vennes et al. (1997).
Table 1. Radial velocities used for computing the orbit of the IP Eri system.

\begin{tabular}{lc}
\hline \hline HJD & $V_{r}\left(\mathrm{~km} \mathrm{~s}^{-1}\right)$ \\
\hline 2455046.711 & 11.17 \\
2455066.748 & 11.47 \\
2455080.698 & 11.70 \\
2455132.581 & 12.54 \\
2455201.387 & 13.40 \\
2455218.405 & 13.60 \\
2455421.686 & 16.16 \\
2455497.538 & 16.80 \\
2455572.383 & 17.13 \\
2455936.430 & 12.65 \\
2455953.412 & 12.04 \\
2455966.392 & 11.75 \\
2455967.331 & 11.83 \\
2456131.726 & 11.39 \\
2456199.603 & 12.34 \\
2456247.567 & 13.13 \\
2456309.336 & 13.95 \\
2456332.371 & 14.33 \\
\hline
\end{tabular}

Notes. Uncertainties on the radial velocities are about $40 \mathrm{~m} \mathrm{~s}^{-1}$.

\section{Orbital elements}

The 18 high-resolution spectra used to compute the spectroscopic orbital elements were obtained with the HERMES/Mercator spectrograph (Raskin et al. 2011), operating at an average resolution of 85000 in high-resolution mode and with a spectral range of [ $\lambda 370-900 \mathrm{~nm}]$. The spectra were reduced with the HERMES pipeline, and the radial velocities, computed by cross-correlating the observed spectra with an Arcturus template, are on the IAU wavelength system defined by the standards from Udry et al. (1999a,b). The individual radial velocities $V_{r}$ are listed in Table 1 . The errors are dominated by the drift of the air refractive index caused by the atmopsheric pressure variations in the spectrograph room (see Fig. 9 in Raskin et al. 2011). The long-term stability during the 4 years of operations of the HERMES spectrograph turns out to be $\sim 40 \mathrm{~m} \mathrm{~s}^{-1}$ as derived from the standard deviation of the radial velocities of the monitored IAU standards. This may be considered as the precision on the radial velocities produced by HERMES.

The corresponding orbital solution is listed in Table 2 and displayed in Fig. 2. The uncertainties of about $30 \mathrm{~m} \mathrm{~s}^{-1}$ on the orbital parameters $V_{\gamma}$ and $K_{1}$ are consistent with the precision of the spectrograph. Our orbital solution does not include the radial-velocity measurement $V_{r}=14.94 \pm 0.10 \mathrm{~km} \mathrm{~s}^{-1}$ obtained by Chubak et al. (2012) on JD 2455261 , but we checked a posteriori that it falls on the orbital solution within $0.1 \mathrm{~km} \mathrm{~s}^{-1}$. It is worth mentioning that IP Eri was imaged using the HST by Barstow et al. (2001) and the binary was not resolved, setting an upper limit on the orbital period of about $19 \mathrm{yr}$, consistent with the 2.9-yr period found here. No orbit could be adjusted to the $\mathrm{O}-\mathrm{C}$ residuals of the IP Eri system (see lower panel of Fig. 2) when testing for the presence of a hypothetical third component.

The current mass function of the system $f\left(M_{1}, M_{2}\right)=$ $0.0036 M_{\odot}$ constrains the primary mass $M_{1}$ to be lower than 4.27 $M_{\odot}$, if $M_{2}=0.43 M_{\odot}$ for the He-WD (see Sect. 2). 
Table 2. Spectroscopic orbital elements of IP Eri.

\begin{tabular}{lrll}
\hline \hline$\omega\left(^{\circ}\right)$ & 128.3 & \pm & 2.0 \\
$e$ & 0.25 & \pm & 0.01 \\
$P(\mathrm{~d})$ & 1071.0 & \pm & 1.8 \\
$T_{0}(\mathrm{JD})$ & 2455956.9 & \pm & 4.7 \\
$V_{\gamma}\left(\mathrm{km} \mathrm{s}^{-1}\right)$ & +14.59 & \pm & 0.03 \\
$K_{1}\left(\mathrm{~km} \mathrm{~s}^{-1}\right)$ & 3.30 & \pm & 0.03 \\
$f(M)\left(M_{\odot}\right)$ & 0.0036 & \pm & 0.0001 \\
$a_{1} \sin i(\mathrm{Gm})$ & 47.13 & & \\
$N$ & 18 & & \\
$\sigma(\mathrm{O}-\mathrm{C})\left(\mathrm{km} \mathrm{s}^{-1}\right)$ & 0.06 & & \\
\hline
\end{tabular}

\section{Atmospheric parameters}

To derive the atmospheric parameters and the detailed abundances, we used two spectra with high signal-to-noise ratios from the list of Table 1 (namely those obtained on August 22 and September 5, 2009, respectively HJD 2455066.748 and 2455 080.698). The first guesses for the atmospheric parameters were determined from the photometry. The color indices $J-K=0.62$ and $V-K=2.23$ yield a first estimate of $T_{\text {eff }}=4900 \mathrm{~K}$ for the effective temperature, using the calibrations of Bessell et al. (1998), in perfect agreement with previous estimates. From the calibration of MK spectral types provided by Cox (2000), we adopted $\log g=2.1$ as initial guess for the surface gravity of a giant star of spectral type K0 (despite the fact that the luminosity class suggests a higher gravity). We initially assumed the metallicity to be solar.

The atmospheric parameters were then determined iteratively using the BACCHUS pipeline developed by one of the author (TMa; see also Jofre et al. 2014) in the context of the Gaia-ESO survey (Gilmore et al. 2012). This pipeline is based on the 1D LTE spectrum-synthesis code Turbospectrum (Alvarez \& Plez 1998; Plez 2012) and allows an automated determination of effective temperature $T_{\text {eff }}$, surface gravity $\log g$, metallicity $[\mathrm{Fe} / \mathrm{H}]$ and microturbulent velocity $\xi$. We used MARCS model atmospheres (Gustafsson et al. 2008) along with a selection of neutral and singly ionized Fe lines that have been selected for the analysis of stellar spectra in the framework of the Gaia-ESO survey. Oscillator strengths are from the VALD database (Kupka et al. 2000). The classical method to obtain the atmospheric parameters consists in avoiding trends in the $[\mathrm{Fe} / \mathrm{H}]$ vs. $\chi$ and $[\mathrm{Fe} / \mathrm{H}]$ vs. $W / \lambda$ relations (where $\chi$ is the lower excitation energy of the considered line, $W$ its measured equivalent width and $\lambda$ its wavelength) but also in forcing lines of Fe I and Fe II to yield the same abundance (see Fig. 3).

The equivalent widths are automatically measured using spectrum synthesis with the atmospheric parameters determined at the previous iteration. The synthetic spectra were convolved with a Gaussian function with full width at half maximum of $6.5 \mathrm{~km} \mathrm{~s}^{-1}$. Only iron lines having reduced equivalent widths $(W / \lambda)$ lower than $0.025 \mathrm{~m} \AA / \AA$ were kept in the analysis. We thus obtain the following atmospheric parameters: $T_{\mathrm{eff}}=4960 \pm$ $100 \mathrm{~K}, \log g=3.3 \pm 0.3,[\mathrm{Fe} / \mathrm{H}]=+0.09 \pm 0.08$ and $\xi=$ $1.5 \pm 0.1 \mathrm{~km} \mathrm{~s}^{-1}$. We used the solar reference values from Grevesse et al. (2007) where $A_{\odot}(\mathrm{Fe})=7.45$.

\section{Abundances}

The detailed abundance analysis was performed (in the framework of Local Thermodynamical Equilibrium - LTE) using the abundance module of the BACCHUS pipeline. The selection of

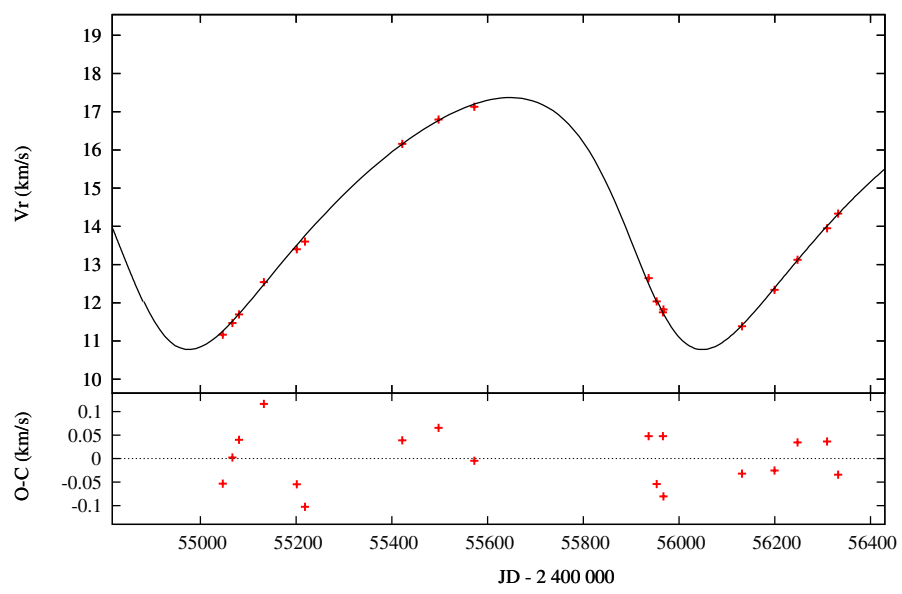

Fig. 2. The orbital solution for IP Eri. The lower panel shows the observed $(\mathrm{O})$ minus the calculated $(\mathrm{C})$ orbits.

atomic and molecular lines was performed over the whole wavelength range covered by the HERMES/Mercator spectrograph. The atomic line list used for the detailed analysis is given in Appendix A. It includes the isotopic shifts for Ba II (with an update for isotopes 130 and 132) and the hyperfine structure for La II from Masseron (2006). The $\mathrm{CH}$ molecular line list is from Masseron et al. (2014). The references for the other molecular line lists ( $\mathrm{TiO}, \mathrm{SiO}, \mathrm{VO}, \mathrm{C}_{2}, \mathrm{CN}, \mathrm{NH}, \mathrm{OH}, \mathrm{MgH}, \mathrm{SiH}, \mathrm{CaH}$ and $\mathrm{FeH}$ ) can be found in Gustafsson et al. (2008). Line fitting is essentially based on a least-square minimization method and all lines are visually inspected to check for possible bad fits (due to, e.g., line blends, cosmic hits, ...). The results of the detailed abundance analysis are presented in Table 3.

\section{1. $C, N, O$ and ${ }^{12} C /{ }^{13} C$}

The numerous neutral carbon atomic lines lead to an abundance of $[\mathrm{C} / \mathrm{Fe}]=0.08 \pm 0.16 \mathrm{dex}$. The nitrogen abundance is determined from numerous molecular $\mathrm{CN}$ lines selected over a large spectral range $[\lambda 640-890 \mathrm{~nm}]$. An example of the fit of $\mathrm{CN}$ lines in the $[\lambda 812.2-813.2 \mathrm{~nm}]$ region is shown in Fig. 4: synthetic spectra with $[\mathrm{N} / \mathrm{Fe}]=-0.12 \pm 0.3 \mathrm{dex}$ (see Table 3 ) are compared with the observed spectrum of IP Eri. The oxygen abundance is derived from the sole [O I] $\lambda 630.03 \mathrm{~nm}$ line which is supposed to be free from NLTE effects (Asplund 2005). Another forbidden line at $\lambda 636.38 \mathrm{~nm}$ is in the red wing of a Ca I autoionization line which is difficult to fit in our spectrum. The oxygen triplet at $\lambda 777.19,777.42$ and $777.54 \mathrm{~nm}$ gives an abundance $A(\mathrm{O}) \approx 9.46 \pm 0.07 \mathrm{dex}$, in disagreement by about $0.7 \mathrm{dex}$ with respect to the $\lambda 630.03 \mathrm{~nm}$ line. This discrepancy is mainly due to the NLTE effect of diffusion in the triplet as clearly explained by Asplund (2005). No OH lines are available in the HERMES spectrum to better constrain the oxygen abundance. The resulting $\mathrm{C} / \mathrm{O}$ ratio is 0.55 , in agreement with the solar value.

The carbon isotopic ratio ${ }^{12} \mathrm{C} /{ }^{13} \mathrm{C}$ is deduced from the ${ }^{12} \mathrm{CN}$ and ${ }^{13} \mathrm{CN}$ molecular lines in the $[\lambda 799.5-801.5 \mathrm{~nm}]$ range (see, e.g., Barbuy et al. 1992; Drake \& Pereira 2008). Specifically, we can compare the ${ }^{12} \mathrm{CN}$ triplet between $\lambda 800.3$ and $\lambda 800.4 \mathrm{~nm}$ with the ${ }^{13} \mathrm{CN}$ feature at $\lambda 800.45 \mathrm{~nm}$, and the ${ }^{12} \mathrm{CN}$ weak line at $\lambda 801.0 \mathrm{~nm}$ with the ${ }^{13} \mathrm{CN}$ very weak line at $\lambda 801.05 \mathrm{~nm}$. The latter is in the far red wing of the former. The high resolution of the HERMES spectrograph is able to separate the two components. The best fit of the entire [ $\lambda 799.5-801.5 \mathrm{~nm}] \mathrm{spec}-$ tral range gives a carbon abundance of $A(C)=8.56 \pm 0.10$ dex, 
A\&A 567, A30 (2014)
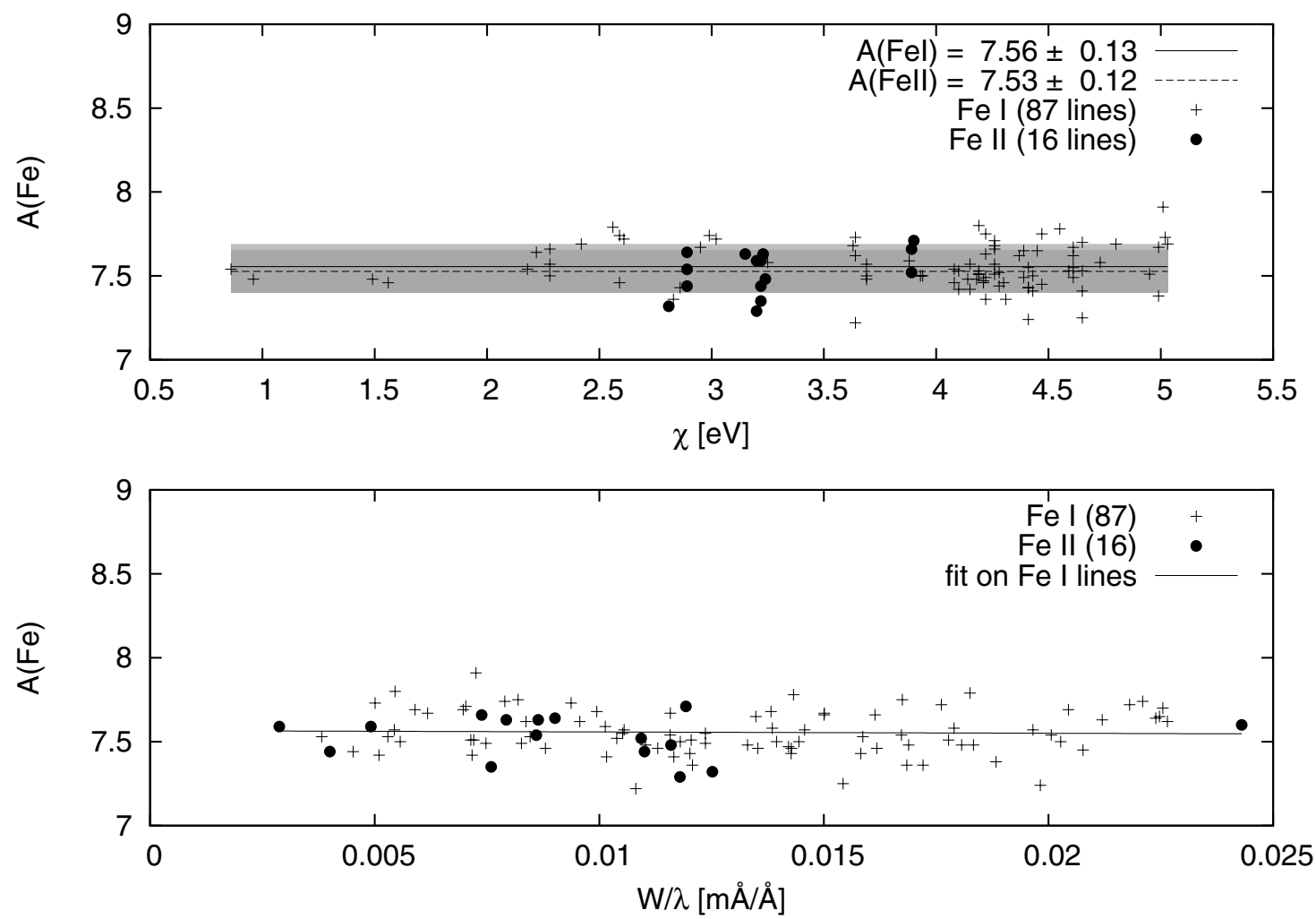

Fig. 3. Line by line abundance analysis for Fe I and Fe II lines as a function of the excitation potential $\chi$ and the reduced equivalent width $W / \lambda$. The grey areas represent the standard deviations around the mean abundances of Fe I and Fe II.

Table 3. Results of the chemical abundance analysis.

\begin{tabular}{|c|c|c|c|c|c|c|c|}
\hline$X$ & & $A(X)$ & {$[X / \mathrm{H}]$} & {$[X / \mathrm{Fe}]$} & $\sigma_{\text {stat }}$ & $N$ & Comments \\
\hline $\mathrm{C}$ & I & 8.56 & 0.17 & 0.08 & 0.16 & 17 & Atomic lines \\
\hline $\mathrm{N}$ & & 7.75 & -0.03 & -0.12 & 0.11 & 447 & $\mathrm{CN}$ lines \\
\hline $\mathrm{O}$ & I & 8.82 & 0.16 & 0.07 & - & 1 & $\lambda 630.03$ \\
\hline $\mathrm{Na}$ & I & 6.45 & 0.28 & 0.19 & 0.11 & 8 & \\
\hline $\mathrm{Mg}$ & I & 7.80 & 0.27 & 0.18 & 0.10 & 10 & \\
\hline $\mathrm{Al}$ & I & 6.67 & 0.30 & 0.21 & 0.04 & 7 & \\
\hline $\mathrm{Si}$ & I & 7.55 & 0.04 & -0.05 & 0.10 & 15 & \\
\hline $\mathrm{Ca}$ & I & 6.59 & 0.28 & 0.19 & 0.04 & 8 & \\
\hline $\mathrm{Ti}$ & I & 5.14 & 0.24 & 0.15 & 0.15 & 9 & \\
\hline $\mathrm{Ti}$ & II & 5.03 & 0.13 & 0.04 & 0.15 & 5 & \\
\hline $\mathrm{Fe}$ & I & 7.56 & 0.11 & & 0.13 & 87 & \\
\hline $\mathrm{Fe}$ & II & 7.53 & 0.08 & & 0.12 & 16 & \\
\hline $\mathrm{Sr}$ & I & 3.10 & 0.18 & 0.09 & 0.04 & 3 & \\
\hline $\mathrm{Y}$ & I & 2.33 & 0.12 & 0.03 & 0.05 & 2 & $\lambda 619.17, \lambda 643.50$ \\
\hline $\mathrm{Y}$ & II & 1.94 & -0.27 & -0.18 & 0.11 & 5 & \\
\hline $\mathrm{Zr}$ & I & 2.89 & 0.31 & 0.22 & 0.18 & 4 & \\
\hline $\mathrm{Zr}$ & II & 2.82 & 0.24 & 0.15 & 0.01 & 2 & $\lambda 444.30, \lambda 535.01$ \\
\hline $\mathrm{Ba}$ & II & 2.44 & 0.27 & 0.18 & 0.04 & 4 & \\
\hline $\mathrm{La}$ & II & 1.17 & 0.04 & -0.05 & 0.14 & 10 & \\
\hline $\mathrm{Ce}$ & II & 1.57 & -0.13 & -0.22 & 0.08 & 4 & \\
\hline $\mathrm{Nd}$ & II & 1.50 & +0.05 & -0.04 & 0.12 & 8 & \\
\hline
\end{tabular}

Notes. $A(X)$ is the abundance of species $X$ in the logarithmic scale where $A(\mathrm{H})=12 . \sigma_{\text {stat }}$ is the line-to-line abundance dispersion. $N$ is the number of lines used for the corresponding species. In the column labelled "comments", wavelengths are expressed in nm.

which confirms the abundance derived from atomic carbon lines. With this value, we can only deduce a lower limit for the carbon isotopic ratio of ${ }^{12} \mathrm{C} /{ }^{13} \mathrm{C} \geq 20$, in accordance with the weakness of the ${ }^{13} \mathrm{CN}$ features.

\subsection{Light elements}

Neutral lines from $\mathrm{Na}, \mathrm{Mg}, \mathrm{Al}, \mathrm{Si}$ and Ca provide reliable abundances with a standard deviation lower than, or of the order of, 0.1 dex. Ca is the most enriched among the investigated $\alpha$-elements, and has the lowest dispersion. The Ti abundance derived from TiI lines is consistent with that of $\mathrm{Mg}$ and $\mathrm{Ca}$, whereas the $\mathrm{Ti}$ abundance derived from $\mathrm{Ti}$ II is 0.1 dex lower but still within the statistical uncertainties. IP Eri is slightly enriched in $\alpha$-elements $([\alpha / \mathrm{Fe}]=0.17 \pm 0.06$ when considering $\mathrm{Mg}, \mathrm{Ca}$ and $\mathrm{Ti}$ ). The iron abundance determination is illustrated in Fig. 3 and is consistent with the derived metallicity.

\section{3. s-process elements}

Abundances for elements from the first two s-process peaks are measurable in IP Eri and their values are listed in Table 3. Examples of synthetic spectra are shown around two second-peak s-process elements (see Figs. 5 and 6) with so$\operatorname{lar}([\mathrm{Ba}, \mathrm{Ce} / \mathrm{Fe}]=0.00)$, enhanced $([\mathrm{Ba}, \mathrm{Ce} / \mathrm{Fe}]=1.00)$ and actual abundances $([\mathrm{Ba} / \mathrm{Fe}]=0.18$ and $[\mathrm{Ce} / \mathrm{Fe}]=-0.22)$. For elements from the first s-process peak, lines of neutral and singly ionized $\mathrm{Y}$ and $\mathrm{Zr}$ are available. Abundances from neutral and ionized species agree within the statistical uncertainties, excepted for Y, with abundances from neutral lines being somewhat larger than those derived from ionized lines. This trend, if real, could be explained by the NLTE mechanism of overionization of the dominant neutral species due to UV radiation of non-local origin as shown, e.g., for Mg by Merle et al. (2011) or for Fe by Lind et al. (2012). These NLTE effects altering the 


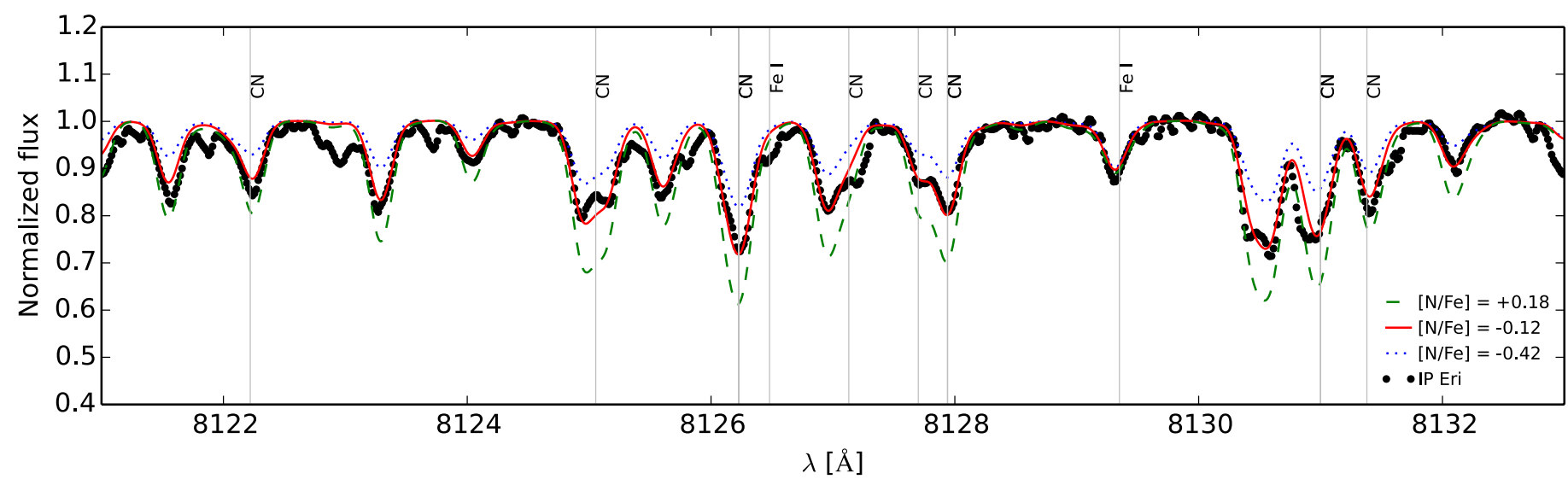

Fig. 4. Example of CN-line fitting.

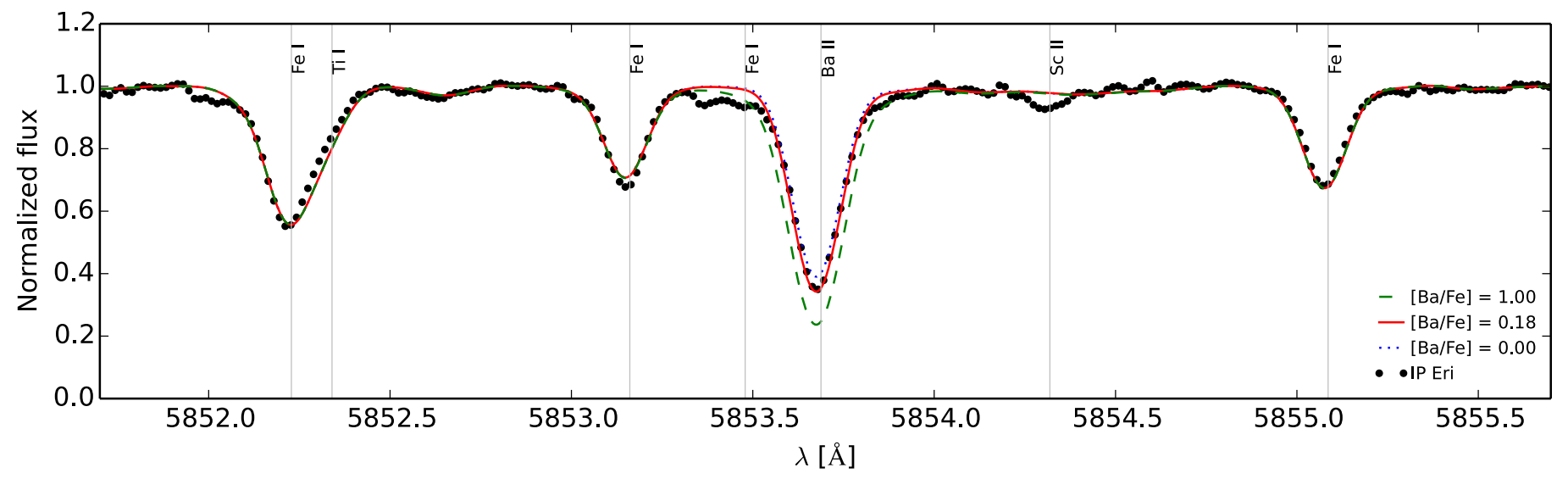

Fig. 5. Example of Ba II line fitting (Ba II $\lambda 585.37 \mathrm{~nm}$ ).

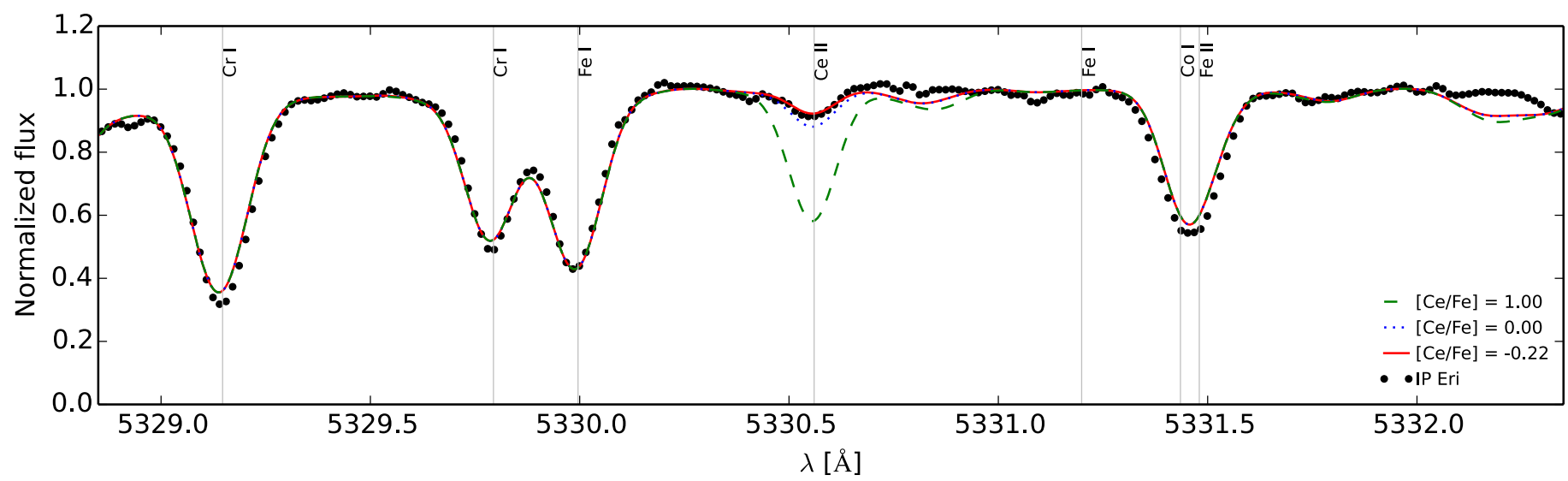

Fig. 6. Example of Ce II line fitting (Ce II $\lambda 533.01 \mathrm{~nm})$.

ionization equilibrium of s-process elements should be investigated, but such an analysis is beyond the scope of this paper.

An average light-s-process abundance of $[1 \mathrm{~s} / \mathrm{Fe}]=0.06 \pm$ 0.04 is obtained, based on SrI, Y II, and Zr II abundances, as compared to $[\mathrm{hs} / \mathrm{Fe}]=-0.03 \pm 0.05$, based on Ba II, La II, and Ce II abundances. The subsolar Ce II abundance is supported by a similarly subsolar $\mathrm{Nd}$ abundance, as derived from $\mathrm{Nd}$ II lines. Thus, there does not seem to be a significant s-process enrichment in IP Eri. This issue is discussed further in Sect. 6, where the IP Eri abundances are confronted with abundances in barium stars and with expectations from the chemical evolution of the Galaxy.

\section{Confrontation with normal field stars and barium stars}

We have compared the abundances of IP Eri with those of field stars and with those from a sample of mild barium stars, to confirm that the abundances of s-process elements in IP Eri derived in Sect. 5.3 are by no means peculiar.

We first consider the light-element abundances $[X / \mathrm{Fe}]$ in IP Eri which are compared in Fig. 7 with abundances in a large sample of $\mathrm{F}$ and $\mathrm{G}$ stars from the galactic disc (Edvardsson et al. 1993). We corrected for the zero-point solar abundance offset, since Edvardsson et al. (1993) used a different reference value of $A_{\odot}(\mathrm{Fe})=7.51$. Figure 7 shows that the light-element 


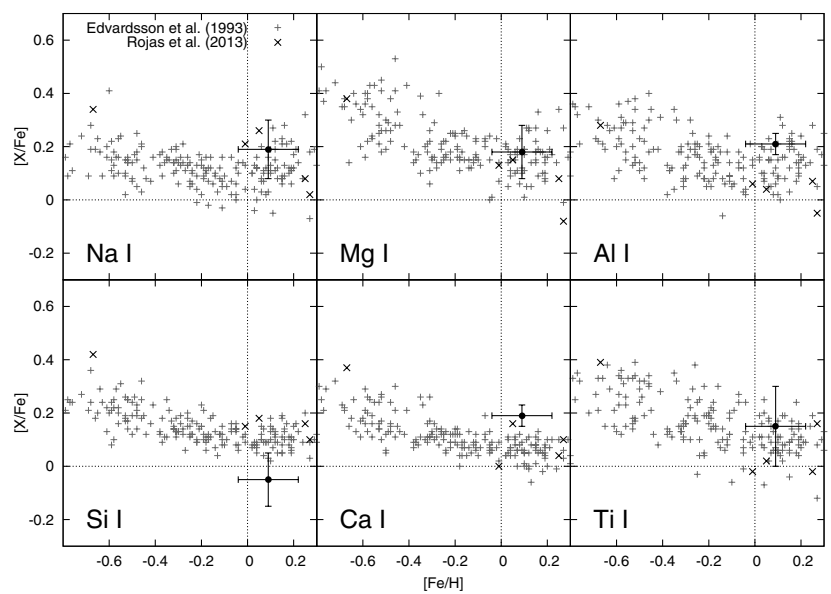

Fig. 7. Comparison of light-element abundances in IP Eri (black dot), in field stars (Edvardsson et al. 1993, grey plusses), and in mild barium stars (Rojas et al. 2013, black crosses).

abundances in IP Eri are in relatively good agreement with those of moderately metal-rich stars. The $\mathrm{Na}$ I, Mg I, Al I and Ti I abundances of IP Eri are located within the abundance distribution of the galactic sample. O I (not shown in Fig. 7) and Ca I are slightly more abundant as compared to the disc stars. The oxygen overabundance may be trusted though, since Edvardsson et al. (1993) used a scaling relation to transform abundances from the high excitation lines that they used $(\lambda 615.8 \mathrm{~nm}$ as well as the $\lambda 777.3 \mathrm{~nm}$ triplet) to the abundance from the [OI] $\lambda 630.0 \mathrm{~nm}$ line that we used. For Ca, there are no lines in common with the Edvardsson et al. analysis. We used saturated and strong lines which are affected by NLTE effects, as shown by Mashonkina et al. (2007). These authors predict positive NLTE abundance corrections (between 0 and 0.07 dex for a model with $T_{\text {eff }}=5000 \mathrm{~K}, \log g=3$ and $[\mathrm{Fe} / \mathrm{H}]=0$ ), which, if accounted for, would further strengthen the $\mathrm{Ca}$ enrichment as compared to the Edvardsson et al. (1993) values for field stars. We have also compared IP Eri light-element abundances with those of a sample of mild barium stars from Rojas et al. (2013). Their lightelement abundances are similar to those of field stars.

On the contrary, the Si I abundance of IP Eri (for which most of the lines used are common to the two studies) is lower than the value derived for the disc-star distribution. The IP Eri abundance of TiI matches the average abundance of the disc stars of similar metallicities, but our value suffers from large error bars due to a larger set of lines in our analysis ( 9 against 4 for Edvardsson et al. 1993). This large dispersion may be partially explained by the fact that all the lines have equivalent widths larger than $150 \mathrm{~m} \AA$ and are consequently less sensitive to abundance changes.

The s-process element abundances in IP Eri are compared with those of disc stars in Fig. 8. The comparison sample is from Edvardsson et al. (1993) for Y II, Reddy et al. (2003) for Sr I and Ce II, Pompéia et al. (2011) for Zr II, Ba II and La II. The slight enrichment in $\mathrm{Zr}$ II and Ba II observed in IP Eri and is typical of the enrichment trend observed for disc stars. Y II and Ce II appear peculiar in that they are underabundant in IP Eri as compared to disc stars of similar metallicities.

For Sr I, the only abundances available for comparison are from Reddy et al. (2003). They are based on a single line, and unfortunately, the Reddy et al. sample includes only stars with solar and sub-solar metallicities, giving the false impression that the IP Eri abundances are discrepant. For La II, data

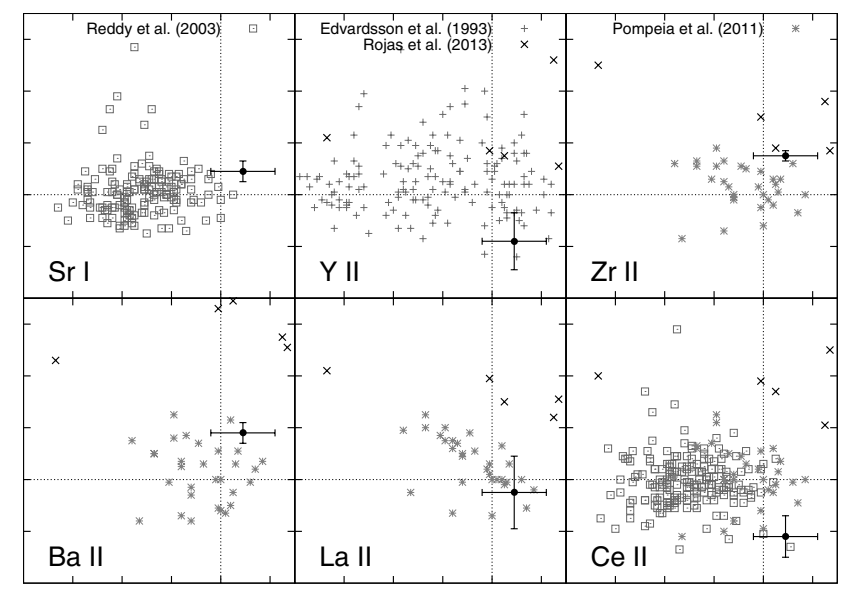

Fig. 8. Comparison of s-process element abundances of IP Eri with field stars (Edvardsson et al. 1993, grey plusses; Reddy et al. 2003, grey squares; Pompéia et al. 2011, grey asterisks) and mild barium stars (Rojas et al. 2013, black crosses).

from Pompéia et al. (2011) are the only ones available for comparison, and the slight La II underabundance (with respect to the Sun) observed in IP Eri matches the trend observed among disc stars.

The comparison with the sample of mild barium stars of Rojas et al. (2013) is not discriminating for first-peak s-process elements (top panels of Fig. 8). But abundances from secondpeak s-process elements in IP Eri are clearly lower than those of mild barium stars (bottom panels of Fig. 8). From these comparisons, we conclude that the slight enrichment in $\alpha$ and s-process elements in IP Eri is largely consistent with the chemical evolution of the Galaxy, so that there is no obvious signature from a chemical pollution resulting from mass transfer.

\section{The evolutionary context of IP Eri}

He WDs form when a star loses its hydrogen-rich envelope before it ignites helium. For a single star, this is not possible within a Hubble time since only stars with masses $M \lesssim$ $0.45-0.5 M_{\odot}$ can avoid helium ignition. A binary scenario is therefore required.

As illustrated in Fig. 9, different evolutionary channels can account for these objects. The first one involves mass transfer by Roche lobe overflow (RLOF). The long period of IP Eri (1071 d) imposes that mass transfer starts while the star is already on the red-giant branch (late case B). Because of the presence of a deep convective envelope in the donor, two outcomes are possible depending on the mass ratio $q=M_{\text {donor }} / M_{\text {gainer }}$. If $q>1.3-1.5$ (Soberman et al. 1997; Hurley et al. 2002), the mass transfer is dynamically unstable; after a rapid stage of common-envelope evolution, a short-period system forms (channel Ia in Fig. 9). In the alternative configuration $(q<1.3-1.5)$, soon after RLOF starts, the mass ratio reverses and subsequent mass transfer leads to the expansion of the orbit. The outcome is then a long-period system similar to IP Eri (channel Ib). However, in this RLOF scenario, tidal interactions are very strong because of the extended convective envelope of the Roche-filling donor star and the orbit always circularizes. This channel thus cannot explain the high eccentricity of IP Eri.

A solution to the eccentricity problem has been described in a companion paper (Siess et al. 2014). Based on binary-evolution calculations with the code BINSTAR (Siess et al. 2013; Davis et al. 2013; Deschamps et al. 2013), we showed that if the 


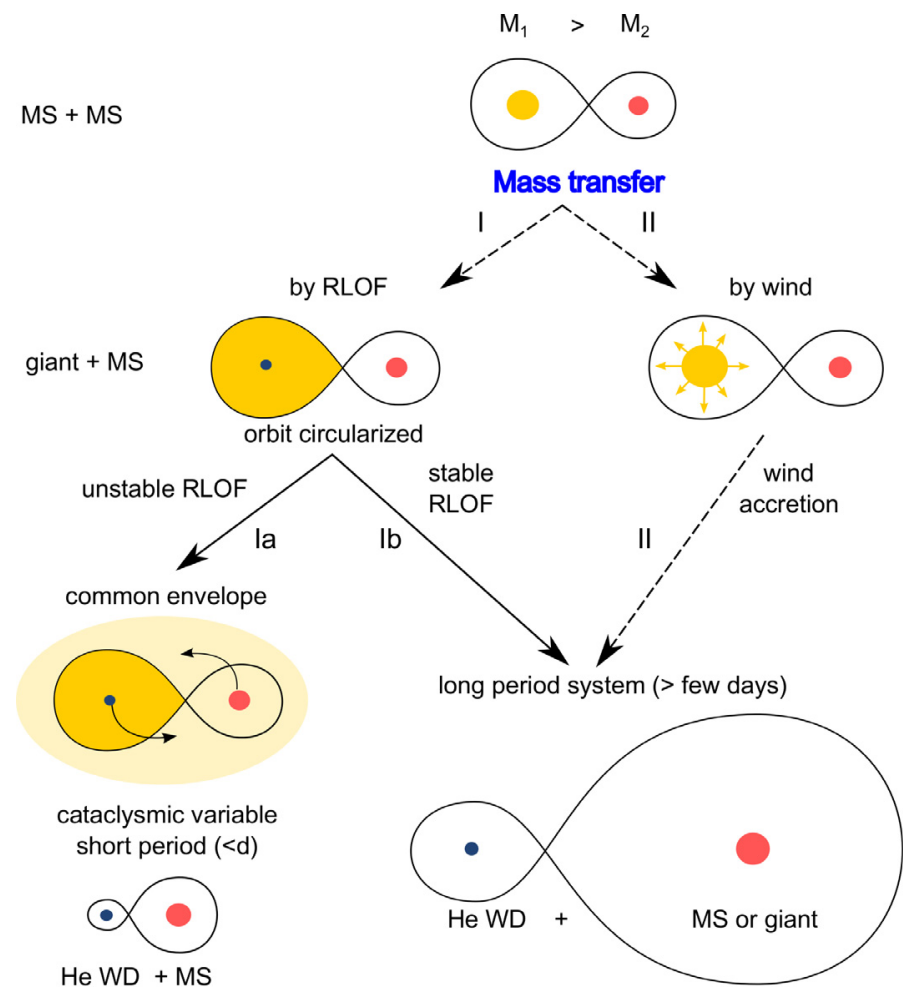

Fig. 9. Evolutionary channels for the formation of a He WD. The dashed lines refer to channels where the eccentricity can be preserved (see text for details).

envelope of the He-WD progenitor is lost via tidally-enhanced winds (channel II), the circularization can be avoided. Tout \& Eggleton (1988) suggested that the presence of a companion star can substantially increase the mass-loss rate of the evolved component. In this situation, the donor star loses its envelope while remaining inside its Roche potential and tidal forces are significantly reduced. Moreover, if the system has an initial eccentricity, the orbital wind mass-transfer modulation (Soker 2000) provides an eccentricity-pumping mechanism that counteracts the tidal circularization. We showed that such a scenario is able to account for all the orbital properties of a system like IP Eri.

In Fig. 10 we compare the orbital properties of IP Eri with those of pre- ( $\mathrm{K}$ giants in open clusters from Mermilliod et al. 2007 and binary M giants from Famaey et al. 2009; Jorissen et al. 2009) and post-mass-transfer binaries (barium and S stars from Jorissen et al. 1998, updated with some recently published orbits from Gorlova et al. 2014). We also show in the $e-\log P$ diagram the location of the G5 IV + WD system HR 1608 (=63 Eri). This system has an eccentricity $(e=0.30 \pm 0.06)$ and a pe$\operatorname{riod}(P=903 \pm 5 \mathrm{~d})$ very similar to those of IP Eri (from Beavers \& Eitter 1988, quoted by Landsman et al. 1993). The revised HIPPARCos parallax $(18.53 \pm 0.84$ mas; van Leeuwen 2007) yields a distance range $51-56 \mathrm{pc}$ for HR 1608 , which implies a WD mass around $0.4 M_{\odot}$ (Landsman et al. 1993), and thus a He-WD. A more recent analysis of the WD parameters by Vennes et al. (1998) suggests instead a higher mass range $\left(0.51-0.67 M_{\odot}\right)$. We also include in Fig. 10 the sdB binaries with long periods (black crosses) from Østensen \& Van Winckel (2011, 2012), Vos et al. (2012, 2013), Deca et al. (2012), and Barlow et al. (2012, 2013).

IP Eri and HR 1608 have the largest eccentricities when compared to the long-period sdB systems, a fact whose significance is difficult to assess with so few He-WD systems. What

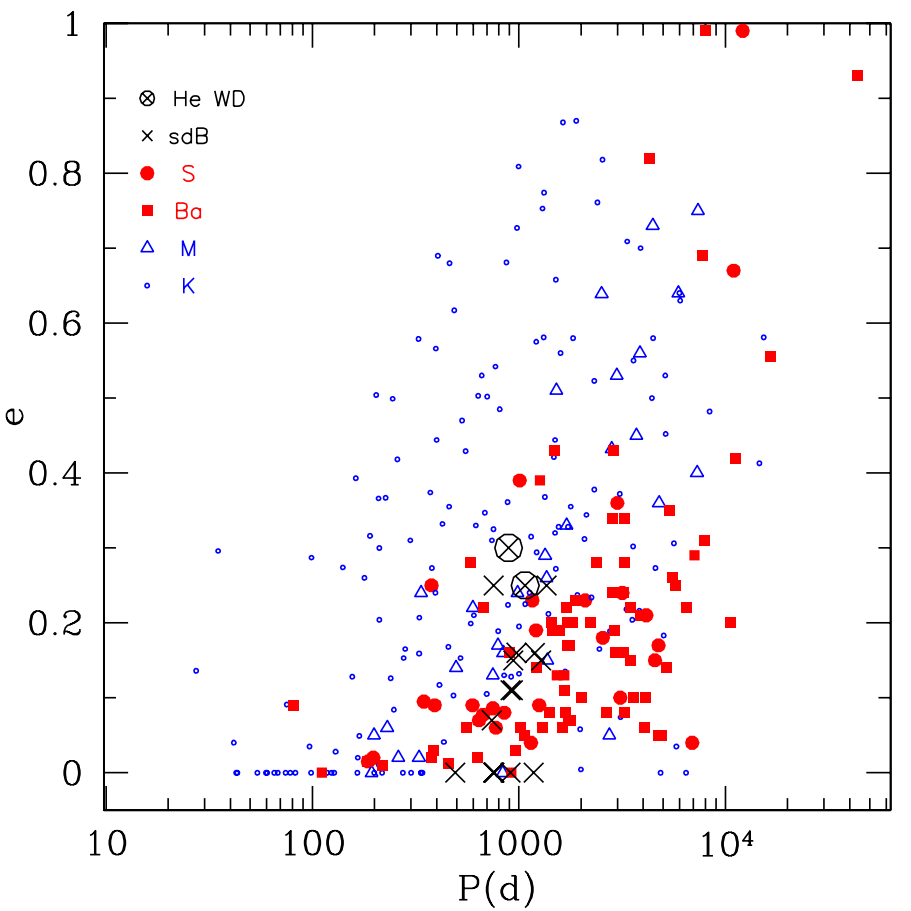

Fig. 10. The positions of IP Eri and its twin system HR 1608 (black circled crosses; Beavers \& Eitter 1988, quoted by Landsman et al. 1993) in the eccentricity - period diagram are compared with those of the long-period sdB binaries, with (mostly) pre-mass-transfer binaries (normal $\mathrm{K}$ and $\mathrm{M}$ giants), and with post-mass-transfer binaries (Barium and $\mathrm{S}$ stars). Symbols are as indicated in the figure label.

seems significant, however, is the fact that (long-period) sdB and He-WD systems occupy a rather restricted period range around $10^{3} \mathrm{~d}$. In that respect, they differ from the barium and $\mathrm{S}$ binaries, two other families of post-mass-transfer systems, which spread over a more extended period range. This difference might be related to the fact that $\mathrm{sdB}$ and $\mathrm{He}-\mathrm{WD}$ binaries are the end products of mass transfer occurring on the first red giant branch, whereas barium and $\mathrm{S}$ systems involved mass transfer on the AGB.

\section{Summary}

IP Eri is an unusual long-period binary system with a high eccentricity. Our abundance analysis reveals that it is not enriched in s-process elements, giving additional support to He-WD nature of the hot component that avoided evolution along the AGB. The abundances of the light elements do not reveal any peculiarities and within the error bars, the abundances are very close to solar. What is more surprising is that IP Eri alike its twin HR 1608 have the largest eccentricities among their closely related $\mathrm{sdB}$ systems and are very concentrated around a thousand-day period, which is likely related to the fact that the donor stars lose their envelope on the red-giant branch. However, the statistics remains small and additional data are highly desirable in order to confirm the formation channel of these systems.

Acknowledgements. This work has been partly funded by an Action de recherche concertée (ARC) from the Direction générale de l'Enseignement non obligatoire et de la Recherche scientifique - Direction de la recherche scientifique - Communauté française de Belgique. T.M. is supported by the FNRS-F.R.S. as temporary post-doctoral researcher under grant No. 2.4513.11. The Mercator telescope is operated thanks to grant number G.0C31.13 of the FWO under the Big Science initiative of the Flemish governement. Based 
on observations obtained with the HERMES spectrograph, supported by the Fund for Scientific Research of Flanders (FWO), the Research Council of K.U. Leuven, the Fonds National de la Recherche Scientifique (F.R.S.-FNRS), Belgium, the Royal Observatory of Belgium, the Observatoire de Genève, Switzerland and the Thüringer Landessternwarte Tautenburg, Germany.

\section{References}

Alvarez, R., \& Plez, B. 1998, A\&A, 330, 1109 Asplund, M. 2005, ARA\&A, 43, 481

Bailer-Jones, C. A. L. 2011, MNRAS, 411, 435

Barbuy, B., Jorissen, A., Rossi, S. C. F., \& Arnould, M. 1992, A\&A, 262, 216

Barlow, B. N., Wade, R. A., Liss, S. E., Østensen, R. H., \& Van Winckel, H. 2012, ApJ, 758, 58

Barlow, B. N., Liss, S. E., Wade, R. A., \& Green, E. M. 2013, ApJ, 771, 23

Barstow, M. A., Bond, H. E., Burleigh, M. R., \& Holberg, J. B. 2001, MNRAS, 322,891

Beavers, W. I., \& Eitter, J. J. 1988, in BAAS, 20, 737

Bessell, M. S., Castelli, F., \& Plez, B. 1998, A\&A, 333, 231

Bidelman, W. P., \& Keenan, P. C. 1951, ApJ, 114, 473

Bowyer, S., Lieu, R., Lampton, M., et al. 1994, ApJS, 93, 569

Bowyer, S., Lampton, M., Lewis, J., et al. 1996, ApJS, 102, 129

Burleigh, M. R., Barstow, M. A., \& Fleming, T. A. 1997, MNRAS, 287, 381

Chubak, C., Marcy, G., Fischer, D. A., et al. 2012 [arXiv: 1207. 6212]

Cox, A. N. 2000, Allen's astrophysical quantities (Springer)

Cutispoto, G., Pallavicini, R., Kuerster, M., \& Rodono, M. 1995, A\&A, 297, 764

Cutispoto, G., Pastori, L., Tagliaferri, G., Messina, S., \& Pallavicini, R. 1999, A\&AS, 138, 87

Cutri, R. M., Skrutskie, M. F., van Dyk, S., et al. 2003, VizieR Online Data Catalog: II/246

Davis, P. J., Siess, L., \& Deschamps, R. 2013, A\&A, 556, A4

Deca, J., Marsh, T. R., Østensen, R. H., et al. 2012, MNRAS, 421, 2798

Deschamps, R., Siess, L., Davis, P. J., \& Jorissen, A. 2013, A\&A, 557, A40

Drake, N. A., \& Pereira, C. B. 2008, AJ, 135, 1070

Driebe, T., Schoenberner, D., Bloecker, T., \& Herwig, F. 1998, A\&A, 339, 123

Edvardsson, B., Andersen, J., Gustafsson, B., et al. 1993, A\&A, 275, 101

Famaey, B., Pourbaix, D., Frankowski, A., et al. 2009, A\&A, 498, 627

Gilmore, G., Randich, S., Asplund, M., et al. 2012, The Messenger, 147, 25

Gorlova, N., Van Winckel, H., Vos, J., et al. 2014, Setting a new standard in the analysis of binary stars, ed. A. Tkachenko, EAS Conf. Ser. [arXiv: 1403.2287]

Grevesse, N., Asplund, M., \& Sauval, A. J. 2007, Space Sci. Rev., 130, 105 Gustafsson, B., Edvardsson, B., Eriksson, K., et al. 2008, A\&A, 486, 951

Heber, U. 2009, ARA\&A, 47, 211

Hurley, J. R., Pols, O. R., \& Tout, C. A. 2000, MNRAS, 315, 543

Hurley, J. R., Tout, C. A., \& Pols, O. R. 2002, MNRAS, 329, 897

Jofre, P., Heiter, U., Soubiran, C., et al. 2014, A\&A, 564, A133

Jorissen, A., Van Eck, S., Mayor, M., \& Udry, S. 1998, A\&A, 332, 877

Jorissen, A., Frankowski, A., Famaey, B., \& van Eck, S. 2009, A\&A, 498, 489
Kupka, F. G., Ryabchikova, T. A., Piskunov, N. E., Stempels, H. C., \& Weiss, W. W. 2000, Baltic Astron., 9, 590

Landsman, W., Simon, T., \& Bergeron, P. 1993, PASP, 105, 841

Lind, K., Bergemann, M., \& Asplund, M. 2012, MNRAS, 427, 50

Malina, R. F., Marshall, H. L., Antia, B., et al. 1994, AJ, 107, 751

Mashonkina, L., Korn, A. J., \& Przybilla, N. 2007, A\&A, 461, 261

Masseron, T. 2006, Ph.D. Thesis, Observatoire de Paris

Masseron, T., Plez, B., Van Eck, S., et al. 2014, A\&A, submitted

Merle, T., Thévenin, F., Pichon, B., \& Bigot, L. 2011, MNRAS, 418, 863

Mermilliod, J.-C., Andersen, J., Latham, D. W., \& Mayor, M. 2007, A\&A, 473, 829

Østensen, R. H., \& Van Winckel, H. 2011, in Evolution of Compact Binaries, eds. L. Schmidtobreick, M. R. Schreiber, \& C. Tappert, ASP Conf. Ser., 447, 171

Østensen, R. H., \& Van Winckel, H. 2012, in Fifth Meeting on Hot Subdwarf Stars and Related Objects, eds. D. Kilkenny, C. S. Jeffery, \& C. Koen, ASP Conf. Ser., 452, 163

Plez, B. 2012, Astrophysics Source Code Library, 5004

Pojmanski, G. 1997, Acta Astron., 47, 467

Pompéia, L., Masseron, T., Famaey, B., et al. 2011, MNRAS, 415, 1138

Pounds, K. A., Allan, D. J., Barber, C., et al. 1993, MNRAS, 260, 77

Pye, J. P., McGale, P. A., Allan, D. J., et al. 1995, MNRAS, 274, 1165

Raskin, G., van Winckel, H., Hensberge, H., et al. 2011, A\&A, 526, A69

Reddy, B. E., Tomkin, J., Lambert, D. L., \& Allende Prieto, C. 2003, MNRAS, 340,304

Rojas, M., Drake, N. A., Pereira, C. B., \& Kholtygin, A. F. 2013, Astrophysics, 56,57

Siess, L., Izzard, R. G., Davis, P. J., \& Deschamps, R. 2013, A\&A, 550, A100

Siess, L., Davis, P. J., \& Jorissen, A. 2014, A\&A, 565, A57

Soberman, G. E., Phinney, E. S., \& van den Heuvel, E. P. J. 1997, A\&A, 327, 620

Soker, N. 2000, A\&A, 357, 557

Strassmeier, K., Washuettl, A., Granzer, T., Scheck, M., \& Weber, M. 2000, A\&AS, 142, 275

Tout, C. A., \& Eggleton, P. P. 1988, MNRAS, 231, 823

Udry, S., Mayor, M., Maurice, E., et al. 1999a, in Precise Stellar Radial Velocities, eds. J. B. Hearnshaw, \& C. D. Scarfe, IAU Colloq., 170, ASP Conf. Ser., 185, 383

Udry, S., Mayor, M., \& Queloz, D. 1999b, in Precise Stellar Radial Velocities, eds. J. B. Hearnshaw, \& C. D. Scarfe, IAU Colloq., 170, ASP Conf. Ser., 185, 367

van Leeuwen, F. 2007, A\&A, 474, 653

Vennes, S., Mathioudakis, M., Doyle, J. G., Thorstensen, J. R., \& Byrne, P. B. 1995, A\&A, 299, L29

Vennes, S., Christian, D. J., Mathioudakis, M., \& Doyle, J. G. 1997, A\&A, 318, L9

Vennes, S., Christian, D. J., \& Thorstensen, J. R. 1998, ApJ, 502, 763

Vos, J., Østensen, R. H., Degroote, P., et al. 2012, A\&A, 548, A6

Vos, J., Østensen, R. H., Németh, P., et al. 2013, A\&A, 559, A54

Pages 9 to 12 are available in the electronic edition of the journal at http://www. aanda.org 
T. Merle et al.: IP Eri

\section{Appendix A: Linelist}

Table A.1. Line list used for determining atmospheric parameters and chemical composition of atomic species.

\begin{tabular}{|c|c|c|c|}
\hline$\lambda[\mathrm{nm}]$ & $\chi[\mathrm{eV}]$ & $\log g f$ & $A(X)$ \\
\hline$C I$ & \multicolumn{3}{|c|}{$A_{\odot}(\mathrm{C})=8.39$} \\
\hline 493.2049 & 7.685 & -1.884 & 8.25 \\
\hline 538.0337 & 7.685 & -1.615 & 8.63 \\
\hline 555.3174 & 8.643 & -2.370 & 8.45 \\
\hline 633.5701 & 8.771 & -2.370 & 8.63 \\
\hline 633.7183 & 8.771 & -2.450 & 8.65 \\
\hline 658.7610 & 8.537 & -1.021 & 8.62 \\
\hline 661.1353 & 8.851 & -1.837 & 8.64 \\
\hline 667.1845 & 8.851 & -1.651 & 8.61 \\
\hline 768.5190 & 8.771 & -1.519 & 8.58 \\
\hline 784.8241 & 8.848 & -1.731 & 8.68 \\
\hline 785.2859 & 8.851 & -1.683 & 8.74 \\
\hline 786.0877 & 8.851 & -1.148 & 8.65 \\
\hline 788.4490 & 8.847 & -1.580 & 8.31 \\
\hline 807.8479 & 8.848 & -1.817 & 8.69 \\
\hline 833.5148 & 7.685 & -0.420 & 8.60 \\
\hline 872.7126 & 1.264 & -8.136 & 8.61 \\
\hline 881.8479 & 9.003 & -1.060 & 8.20 \\
\hline$O I$ & \multicolumn{3}{|c|}{$A_{\odot}(\mathrm{O})=8.66$} \\
\hline 630.0304 & 0.000 & -9.715 & 8.82 \\
\hline $\mathrm{NaI}$ & \multicolumn{3}{|c|}{$A_{\odot}(\mathrm{Na})=6.17$} \\
\hline 449.7657 & 2.104 & -1.560 & 6.36 \\
\hline 498.2814 & 2.104 & -0.950 & 6.38 \\
\hline 568.2633 & 2.102 & -0.706 & 6.25 \\
\hline 568.8205 & 2.104 & -0.450 & 6.45 \\
\hline 615.4226 & 2.102 & -1.547 & 6.53 \\
\hline 616.0747 & 2.104 & -1.246 & 6.48 \\
\hline 818.3255 & 2.102 & 0.230 & 6.60 \\
\hline 819.4824 & 2.104 & 0.490 & 6.54 \\
\hline$M g I$ & \multicolumn{3}{|c|}{$A_{\odot}(\mathrm{Mg})=7.53$} \\
\hline 552.8405 & 4.346 & -0.620 & 7.70 \\
\hline 571.1088 & 4.346 & -1.833 & 7.67 \\
\hline 631.8717 & 5.108 & -2.103 & 7.86 \\
\hline 631.9237 & 5.108 & -2.324 & 7.92 \\
\hline 631.9495 & 5.108 & -2.803 & 7.89 \\
\hline 738.7689 & 5.753 & -1.100 & 7.82 \\
\hline 769.1553 & 5.753 & -0.783 & 7.68 \\
\hline 871.2676 & 5.932 & -1.670 & \multirow[t]{2}{*}{7.80} \\
\hline 871.2689 & 5.932 & -1.370 & \\
\hline 871.7825 & 5.933 & -0.930 & 7.75 \\
\hline 873.6019 & 5.946 & -0.690 & 7.96 \\
\hline 873.6029 & 5.946 & -1.020 & \\
\hline AlI & \multicolumn{3}{|c|}{$A_{\odot}(\mathrm{Al})=6.37$} \\
\hline 555.7063 & 6.143 & -2.104 & 6.63 \\
\hline 669.6023 & 3.143 & -1.569 & 6.69 \\
\hline 669.8673 & 3.143 & -1.870 & 6.73 \\
\hline
\end{tabular}

Notes. The main source for oscillator strengths $(\log g f)$ is VALD. Fitted individual absolute abundances $A(X)$ are also given. The absolute solar abundances $A_{\odot}(X)$ are from Grevesse et al. (2007). Vertical lines in the left margin mean that all transitions contribute to the same line (fine or hyperfine structure). For Ba II, the atomic mass of the contributing isotope is indicated as a superscript to the transition wavelength.
Table A.1. continued.

\begin{tabular}{|c|c|c|c|}
\hline$\lambda[\mathrm{nm}]$ & $\chi[\mathrm{eV}]$ & $\log g f$ & $A(X)$ \\
\hline 783.5309 & 4.022 & -0.649 & 6.68 \\
\hline 783.6134 & 4.022 & -0.494 & 6.71 \\
\hline 877.2865 & 4.022 & -0.170 & 6.64 \\
\hline 877.3896 & 4.022 & -0.161 & 6.75 \\
\hline Si I & \multicolumn{3}{|c|}{$A_{\odot}(\mathrm{Si})=7.51$} \\
\hline 566.5555 & 4.920 & -1.940 & 7.31 \\
\hline 568.4484 & 4.954 & -1.553 & 7.55 \\
\hline 569.0425 & 4.930 & -1.773 & 7.50 \\
\hline 570.1104 & 4.930 & -1.953 & 7.57 \\
\hline 577.2146 & 5.082 & -1.653 & 7.64 \\
\hline 579.3073 & 4.930 & -1.963 & 7.62 \\
\hline 612.5021 & 5.614 & -1.464 & 7.60 \\
\hline 613.1573 & 5.616 & -1.556 & 7.52 \\
\hline 613.1852 & 5.616 & -1.615 & 7.57 \\
\hline 614.2483 & 5.619 & -1.295 & 7.43 \\
\hline 614.5016 & 5.616 & -1.310 & 7.54 \\
\hline 615.5134 & 5.619 & -0.754 & 7.70 \\
\hline 624.4466 & 5.616 & -1.093 & 7.42 \\
\hline 776.0628 & 6.206 & -1.261 & 7.65 \\
\hline 872.8010 & 6.181 & -0.370 & 7.61 \\
\hline $\mathrm{CaI}$ & \multicolumn{3}{|c|}{$A_{\odot}(\mathrm{Ca})=6.31$} \\
\hline 558.8749 & 2.526 & 0.358 & 6.62 \\
\hline 585.7451 & 2.933 & 0.240 & 6.50 \\
\hline 610.2723 & 1.879 & -0.793 & 6.60 \\
\hline 612.2217 & 1.886 & -0.316 & 6.59 \\
\hline 616.2173 & 1.899 & -0.090 & 6.61 \\
\hline 643.9075 & 2.526 & 0.390 & 6.55 \\
\hline 649.3781 & 2.521 & -0.109 & 6.57 \\
\hline 649.9650 & 2.523 & -0.818 & 6.64 \\
\hline Ti I & \multicolumn{3}{|c|}{$A_{\odot}(\mathrm{Ti})=4.90$} \\
\hline 453.4776 & 0.836 & 0.280 & 5.31 \\
\hline 454.8763 & 0.826 & -0.354 & 4.99 \\
\hline 498.1731 & 0.848 & 0.560 & 5.18 \\
\hline 499.9503 & 0.826 & 0.306 & 5.24 \\
\hline 502.4844 & 0.818 & -0.546 & 5.05 \\
\hline 503.9957 & 0.021 & -1.074 & 5.02 \\
\hline 517.3743 & 0.000 & -1.062 & 4.97 \\
\hline 519.2969 & 0.021 & -1.006 & 5.39 \\
\hline 521.0385 & 0.048 & -0.527 & 5.09 \\
\hline \multicolumn{4}{|l|}{ Ti II } \\
\hline 453.3960 & 1.237 & -0.530 & 4.86 \\
\hline 456.3757 & 1.221 & -0.690 & 5.05 \\
\hline 457.1968 & 1.572 & -0.320 & 4.99 \\
\hline 533.6771 & 1.582 & -1.630 & 4.98 \\
\hline 538.1015 & 1.566 & -1.970 & 5.26 \\
\hline $\mathrm{Fe} \mathrm{I}$ & \multicolumn{3}{|c|}{$A_{\odot}(\mathrm{Fe})=7.45$} \\
\hline 480.8148 & 3.251 & -2.690 & 7.58 \\
\hline 496.2572 & 4.178 & -1.182 & 7.48 \\
\hline 499.2785 & 4.260 & -2.350 & 7.71 \\
\hline 505.8496 & 3.642 & -2.830 & 7.73 \\
\hline 524.3776 & 4.256 & -1.050 & 7.51 \\
\hline 525.3021 & 2.279 & -3.840 & 7.50 \\
\hline 529.4547 & 3.640 & -2.760 & 7.62 \\
\hline 529.5312 & 4.415 & -1.590 & 7.55 \\
\hline 537.3709 & 4.473 & -0.760 & 7.45 \\
\hline 537.9574 & 3.694 & -1.514 & 7.48 \\
\hline 538.9479 & 4.415 & -0.410 & 7.24 \\
\hline 539.7618 & 3.634 & -2.528 & 7.68 \\
\hline 539.8279 & 4.445 & -0.630 & 7.65 \\
\hline 541.2784 & 4.434 & -1.716 & 7.41 \\
\hline 541.7033 & 4.415 & -1.580 & 7.43 \\
\hline
\end{tabular}


Table A.1. continued.

\begin{tabular}{|c|c|c|c|}
\hline$\lambda[\mathrm{nm}]$ & $\chi[\mathrm{eV}]$ & $\log g f$ & $A(X)$ \\
\hline 543.6295 & 4.386 & -1.440 & 7.65 \\
\hline 544.1339 & 4.312 & -1.630 & 7.36 \\
\hline 546.6396 & 4.371 & -0.630 & 7.62 \\
\hline 547.3163 & 4.191 & -2.040 & 7.53 \\
\hline 548.3099 & 4.154 & -1.406 & 7.57 \\
\hline 548.7145 & 4.415 & -1.430 & 7.43 \\
\hline 549.1832 & 4.186 & -2.188 & 7.51 \\
\hline 549.4463 & 4.076 & -1.990 & 7.54 \\
\hline 552.2446 & 4.209 & -1.450 & 7.47 \\
\hline 553.9280 & 3.642 & -2.560 & 7.22 \\
\hline 554.3936 & 4.217 & -1.040 & 7.36 \\
\hline 554.9949 & 3.694 & -2.810 & 7.50 \\
\hline 556.0212 & 4.434 & -1.090 & 7.50 \\
\hline 557.7025 & 5.033 & -1.543 & 7.69 \\
\hline 561.8632 & 4.209 & -1.275 & 7.46 \\
\hline 563.3946 & 4.991 & -0.230 & 7.38 \\
\hline 563.8262 & 4.220 & -0.770 & 7.63 \\
\hline 565.1469 & 4.473 & -1.900 & 7.75 \\
\hline 565.2318 & 4.260 & -1.850 & 7.68 \\
\hline 566.1345 & 4.284 & -1.756 & 7.52 \\
\hline 567.9023 & 4.652 & -0.820 & 7.53 \\
\hline 570.5464 & 4.301 & -1.355 & 7.46 \\
\hline 573.1762 & 4.256 & -1.200 & 7.66 \\
\hline 573.2296 & 4.991 & -1.460 & 7.67 \\
\hline 574.1848 & 4.256 & -1.672 & 7.57 \\
\hline 575.2032 & 4.549 & -1.177 & 7.78 \\
\hline 577.5081 & 4.220 & -1.297 & 7.75 \\
\hline 577.8453 & 2.588 & -3.430 & 7.46 \\
\hline 584.9683 & 3.694 & -2.890 & 7.57 \\
\hline 585.5076 & 4.608 & -1.478 & 7.49 \\
\hline 585.8778 & 4.220 & -2.160 & 7.49 \\
\hline 586.1109 & 4.283 & -2.304 & 7.44 \\
\hline 590.5671 & 4.652 & -0.690 & 7.25 \\
\hline 592.7789 & 4.652 & -0.990 & 7.41 \\
\hline 593.0180 & 4.652 & -0.230 & 7.70 \\
\hline 593.4655 & 3.928 & -1.070 & 7.50 \\
\hline 595.6694 & 0.859 & -4.553 & 7.54 \\
\hline 602.7051 & 4.076 & -1.089 & 7.46 \\
\hline 605.6005 & 4.733 & -0.460 & 7.58 \\
\hline 609.3643 & 4.607 & -1.400 & 7.62 \\
\hline 615.1617 & 2.176 & -3.312 & 7.54 \\
\hline 616.5360 & 4.143 & -1.473 & 7.48 \\
\hline 617.3334 & 2.223 & -2.880 & 7.64 \\
\hline 618.7989 & 3.943 & -1.620 & 7.50 \\
\hline 620.0313 & 2.608 & -2.405 & 7.72 \\
\hline 622.6734 & 3.883 & -2.120 & 7.59 \\
\hline 627.0223 & 2.858 & -2.536 & 7.43 \\
\hline 632.2685 & 2.588 & -2.448 & 7.74 \\
\hline 643.6406 & 4.186 & -2.580 & 7.80 \\
\hline 647.5624 & 2.559 & -2.941 & 7.79 \\
\hline 648.1870 & 2.279 & -2.985 & 7.57 \\
\hline 649.8938 & 0.958 & -4.688 & 7.48 \\
\hline 651.8366 & 2.831 & -2.373 & 7.36 \\
\hline 665.3851 & 4.154 & -2.215 & 7.42 \\
\hline 669.9141 & 4.593 & -2.101 & 7.53 \\
\hline 671.0318 & 1.485 & -4.764 & 7.48 \\
\hline 671.3743 & 4.795 & -1.500 & 7.69 \\
\hline 672.5356 & 4.103 & -2.013 & 7.42 \\
\hline 672.6666 & 4.607 & -1.133 & 7.67 \\
\hline 673.9521 & 1.557 & -4.794 & 7.46 \\
\hline 675.0152 & 2.424 & -2.604 & 7.69 \\
\hline 681.0262 & 4.607 & -0.986 & 7.55 \\
\hline 848.1981 & 4.186 & -1.988 & 7.51 \\
\hline 851.5108 & 3.018 & -2.073 & 7.72 \\
\hline 852.7852 & 5.020 & -1.625 & 7.73 \\
\hline
\end{tabular}

Table A.1. continued.

\begin{tabular}{|c|c|c|c|}
\hline$\lambda[\mathrm{nm}]$ & $\chi[\mathrm{eV}]$ & $\log g f$ & $A(X)$ \\
\hline 857.1804 & 5.010 & -1.414 & 7.91 \\
\hline 859.8829 & 4.386 & -1.088 & 7.49 \\
\hline 862.1601 & 2.949 & -2.320 & 7.67 \\
\hline 863.2414 & 4.103 & -2.341 & 7.53 \\
\hline 869.8706 & 2.990 & -3.452 & 7.74 \\
\hline 869.9454 & 4.955 & -0.380 & 7.51 \\
\hline 880.4623 & 2.279 & -3.234 & 7.66 \\
\hline \multicolumn{4}{|l|}{$\mathrm{Fe} I I$} \\
\hline 499.3358 & 2.807 & -3.684 & 7.32 \\
\hline 523.4625 & 3.221 & -2.180 & 7.60 \\
\hline 525.6938 & 2.891 & -4.182 & 7.64 \\
\hline 532.5553 & 3.221 & -3.160 & 7.44 \\
\hline 533.7732 & 3.230 & -3.720 & 7.63 \\
\hline 541.4073 & 3.221 & -3.580 & 7.35 \\
\hline 542.5257 & 3.199 & -3.220 & 7.29 \\
\hline 553.4847 & 3.245 & -2.865 & 7.48 \\
\hline 599.1376 & 3.153 & -3.647 & 7.63 \\
\hline 608.4111 & 3.199 & -3.881 & 7.59 \\
\hline 611.3322 & 3.221 & -4.230 & 7.59 \\
\hline 614.9258 & 3.889 & -2.841 & 7.66 \\
\hline 624.7557 & 3.892 & -2.435 & 7.52 \\
\hline 636.9462 & 2.891 & -4.110 & 7.44 \\
\hline 643.2680 & 2.891 & -3.570 & 7.54 \\
\hline 645.6383 & 3.903 & -2.185 & 7.71 \\
\hline SrI & \multicolumn{3}{|c|}{$A_{\odot}(\mathrm{Sr})=2.92$} \\
\hline 483.2108 & 1.798 & -0.110 & 3.11 \\
\hline 496.2259 & 1.847 & 0.200 & 3.06 \\
\hline 707.0070 & 1.847 & -0.030 & 3.13 \\
\hline$Y I$ & \multicolumn{3}{|c|}{$A_{\odot}(\mathrm{Y})=2.21$} \\
\hline 619.1718 & 0.000 & -0.970 & 2.38 \\
\hline 643.5004 & 0.066 & -0.820 & 2.28 \\
\hline \multicolumn{4}{|l|}{$Y I I$} \\
\hline 490.0120 & 1.033 & 0.103 & 1.84 \\
\hline 520.0406 & 0.992 & -0.570 & 2.01 \\
\hline 520.5724 & 1.033 & -0.193 & 2.00 \\
\hline 528.9815 & 1.033 & -1.850 & 2.03 \\
\hline 679.5414 & 1.738 & -1.030 & 1.80 \\
\hline $\operatorname{ZrI}$ & \multicolumn{3}{|c|}{$A_{\odot}(\mathrm{Zr})=2.58$} \\
\hline 482.8041 & 0.623 & -0.640 & 2.81 \\
\hline 612.7475 & 0.154 & -1.060 & 2.82 \\
\hline 613.4585 & 0.000 & -1.280 & 2.76 \\
\hline 807.0115 & 0.730 & -0.790 & 3.16 \\
\hline \multicolumn{4}{|l|}{$\mathrm{Zr} I I$} \\
\hline 444.2992 & 1.486 & -0.420 & 2.82 \\
\hline 535.0089 & 1.827 & -1.240 & 2.81 \\
\hline Ba II & \multicolumn{3}{|c|}{$A_{\odot}(\mathrm{Ba})=2.17$} \\
\hline $455.3998^{137}$ & 0.000 & -0.666 & \\
\hline $455.3999^{137}$ & 0.000 & -0.666 & \\
\hline $455.4000^{137}$ & 0.000 & -1.064 & \\
\hline $455.4001^{135}$ & 0.000 & -0.666 & \\
\hline $455.4002^{135}$ & 0.000 & -1.064 & \\
\hline $455.4002^{135}$ & 0.000 & -0.666 & \\
\hline $455.4031^{130}$ & 0.000 & 0.140 & \\
\hline $455.4031^{132}$ & 0.000 & 0.140 & \\
\hline $455.4031^{134}$ & 0.000 & 0.140 & 2.42 \\
\hline $455.4032^{136}$ & 0.000 & 0.140 & \\
\hline $455.4033^{138}$ & 0.000 & 0.140 & \\
\hline $455.4048^{135}$ & 0.000 & -0.219 & \\
\hline $455.4050^{135}$ & 0.000 & -0.666 & \\
\hline
\end{tabular}


T. Merle et al.: IP Eri

Table A.1. continued.

\begin{tabular}{|c|c|c|c|}
\hline$\lambda[\mathrm{nm}]$ & $\chi[\mathrm{eV}]$ & $\log g f$ & $A(X)$ \\
\hline $455.4051^{137}$ & 0.000 & -0.219 & \\
\hline $455.4052^{135}$ & 0.000 & -1.365 & \\
\hline $455.4054^{137}$ & 0.000 & -0.666 & \\
\hline $455.4055^{137}$ & 0.000 & -1.365 & \\
\hline $585.3669^{135}$ & 0.604 & -1.967 & \\
\hline $585.3669^{137}$ & 0.604 & -1.967 & \\
\hline $585.3670^{135}$ & 0.604 & -2.113 & \\
\hline $585.3670^{135}$ & 0.604 & -1.909 & \\
\hline $585.3671^{137}$ & 0.604 & -2.113 & \\
\hline $585.3671^{137}$ & 0.604 & -1.909 & \\
\hline $585.3672^{135}$ & 0.604 & -2.113 & \\
\hline $585.3672^{135}$ & 0.604 & -2.511 & \\
\hline $585.3673^{130}$ & 0.604 & -0.909 & \\
\hline $585.3673^{132}$ & 0.604 & -0.909 & \\
\hline $585.3673^{134}$ & 0.604 & -0.909 & \\
\hline $585.3673^{137}$ & 0.604 & -2.113 & 2.43 \\
\hline $585.3673^{135}$ & 0.604 & -1.812 & \\
\hline $585.3673^{137}$ & 0.604 & -2.511 & \\
\hline $585.3674^{136}$ & 0.604 & -0.909 & \\
\hline $585.3675^{135}$ & 0.604 & -1.909 & \\
\hline $585.3675^{135}$ & 0.604 & -1.365 & \\
\hline $585.3675^{137}$ & 0.604 & -1.812 & \\
\hline $585.3675^{138}$ & 0.604 & -0.909 & \\
\hline $585.3676^{137}$ & 0.604 & -1.909 & \\
\hline $585.3676^{137}$ & 0.604 & -1.365 & \\
\hline $585.3680^{135}$ & 0.604 & -1.967 & \\
\hline $585.3682^{137}$ & 0.604 & -1.967 & \\
\hline $614.1708^{135}$ & 0.704 & -0.456 & \\
\hline $614.1708^{135}$ & 0.704 & -1.264 & \\
\hline $614.1709^{135}$ & 0.704 & -2.410 & \\
\hline $614.1709^{137}$ & 0.704 & -1.264 & \\
\hline $614.1709^{137}$ & 0.704 & -0.456 & \\
\hline $614.1710^{137}$ & 0.704 & -2.410 & \\
\hline $614.1711^{130}$ & 0.704 & -0.030 & \\
\hline $614.1711^{132}$ & 0.704 & -0.030 & \\
\hline $614.1711^{134}$ & 0.704 & -0.030 & \\
\hline $614.1712^{136}$ & 0.704 & -0.030 & \\
\hline $614.1713^{135}$ & 0.704 & -0.662 & \\
\hline $614.1713^{138}$ & 0.704 & -0.030 & 2.40 \\
\hline $614.1714^{135}$ & 0.704 & -1.167 & \\
\hline $614.1715^{135}$ & 0.704 & -2.234 & \\
\hline $614.1715^{137}$ & 0.704 & -0.662 & \\
\hline $614.1716^{135}$ & 0.704 & -0.912 & \\
\hline $614.1716^{137}$ & 0.704 & -1.167 & \\
\hline $614.1717^{135}$ & 0.704 & -1.234 & \\
\hline $614.1717^{135}$ & 0.704 & -1.280 & \\
\hline $614.1717^{137}$ & 0.704 & -2.234 & \\
\hline $614.1718^{137}$ & 0.704 & -0.912 & \\
\hline $614.1719^{137}$ & 0.704 & -1.234 & \\
\hline $614.1719^{137}$ & 0.704 & -1.280 & \\
\hline $6496.883^{135}$ & 0.604 & -1.911 & \\
\hline $6496.883^{137}$ & 0.604 & -1.911 & \\
\hline $6496.888^{135}$ & 0.604 & -1.212 & \\
\hline $6496.888^{137}$ & 0.604 & -1.212 & \\
\hline $6496.895^{130}$ & 0.604 & -0.406 & \\
\hline $6496.895^{132}$ & 0.604 & -0.406 & \\
\hline $6496.895^{134}$ & 0.604 & -0.406 & \\
\hline $6496.895^{135}$ & 0.604 & -0.765 & \\
\hline $6496.896^{137}$ & 0.604 & -0.765 & 2.49 \\
\hline $6496.897^{136}$ & 0.604 & -0.406 & \\
\hline $6496.898^{138}$ & 0.604 & -0.406 & \\
\hline
\end{tabular}

Table A.1. continued.

\begin{tabular}{|c|c|c|c|}
\hline$\lambda[\mathrm{nm}]$ & $\chi[\mathrm{eV}]$ & $\log g f$ & $A(X)$ \\
\hline $6496.900^{135}$ & 0.604 & -1.610 & \\
\hline $6496.902^{135}$ & 0.604 & -1.212 & \\
\hline $6496.902^{137}$ & 0.604 & -1.610 & \\
\hline $6496.904^{137}$ & 0.604 & -1.212 & \\
\hline $6496.906^{135}$ & 0.604 & -1.212 & \\
\hline $6496.909^{137}$ & 0.604 & -1.212 & \\
\hline La II & $A_{\odot}(\mathrm{La})$ & $=1.13$ & \\
\hline 408.6695 & 0.000 & -1.266 & \\
\hline 408.6699 & 0.000 & -1.108 & \\
\hline 408.6702 & 0.000 & -1.119 & \\
\hline 408.6705 & 0.000 & -1.292 & \\
\hline 408.6708 & 0.000 & -0.696 & \\
\hline 408.6709 & 0.000 & -1.094 & \\
\hline 408.6710 & 0.000 & -1.790 & 1.04 \\
\hline 408.6711 & 0.000 & -1.468 & \\
\hline 408.6711 & 0.000 & -3.216 & \\
\hline 408.6717 & 0.000 & -1.292 & \\
\hline 408.6719 & 0.000 & -1.119 & \\
\hline 408.6720 & 0.000 & -1.108 & \\
\hline 408.6721 & 0.000 & -1.266 & \\
\hline 455.8457 & 0.321 & -0.970 & 1.15 \\
\hline 457.4860 & 0.173 & -1.08 & 1.24 \\
\hline 492.0965 & 0.126 & -2.261 & \\
\hline 492.0965 & 0.126 & -2.407 & \\
\hline 492.0966 & 0.126 & -2.065 & \\
\hline 492.0966 & 0.126 & -2.078 & \\
\hline 492.0966 & 0.126 & -2.738 & \\
\hline 492.0968 & 0.126 & -1.831 & \\
\hline 492.0968 & 0.126 & -1.956 & \\
\hline 492.0968 & 0.126 & -2.629 & \\
\hline 492.0971 & 0.126 & -1.646 & \\
\hline 492.0971 & 0.126 & -1.895 & 1.32 \\
\hline 492.0971 & 0.126 & -2.650 & \\
\hline 492.0975 & 0.126 & -1.490 & \\
\hline 492.0975 & 0.126 & -1.891 & \\
\hline 492.0975 & 0.126 & -2.760 & \\
\hline 492.0979 & 0.126 & -1.354 & \\
\hline 492.0979 & 0.126 & -1.957 & \\
\hline 492.0979 & 0.126 & -2.972 & \\
\hline 492.0985 & 0.126 & -1.233 & \\
\hline 492.0985 & 0.126 & -2.162 & \\
\hline 492.0985 & 0.126 & -3.375 & \\
\hline 530.3513 & 0.321 & -1.874 & \\
\hline 530.3513 & 0.321 & -2.363 & \\
\hline 530.3514 & 0.321 & -3.062 & \\
\hline 530.3531 & 0.321 & -2.167 & \\
\hline 530.3532 & 0.321 & -2.247 & 1.32 \\
\hline 530.3532 & 0.321 & -2.622 & \\
\hline 530.3546 & 0.321 & -2.366 & \\
\hline 530.3546 & 0.321 & -2.622 & \\
\hline 530.3547 & 0.321 & -2.351 & \\
\hline 579.7565 & 0.244 & -1.360 & 1.33 \\
\hline 588.0633 & 0.235 & -1.830 & 1.11 \\
\hline 632.0376 & 0.173 & -1.520 & 1.04 \\
\hline 639.0455 & 0.321 & -2.012 & \\
\hline 639.0468 & 0.321 & -2.183 & \\
\hline 639.0468 & 0.321 & -2.752 & \\
\hline 639.0479 & 0.321 & -2.570 & \\
\hline 639.0479 & 0.321 & -3.752 & \\
\hline 639.0480 & 0.321 & -2.390 & \\
\hline
\end{tabular}


Table A.1. continued.

\begin{tabular}{lccc}
\hline \hline$\lambda[\mathrm{nm}]$ & $\chi[\mathrm{eV}]$ & $\log g f$ & $A(X)$ \\
\hline 639.0489 & 0.321 & -2.536 & \\
639.0489 & 0.321 & -3.334 & 1.23 \\
639.0490 & 0.321 & -2.661 & \\
639.0496 & 0.321 & -3.100 & \\
639.0497 & 0.321 & -2.595 & \\
639.0498 & 0.321 & -3.079 & \\
639.0502 & 0.321 & -2.954 & \\
639.0503 & 0.321 & -2.778 & \\
639.0506 & 0.321 & -2.857 & \\
& & & \\
677.4268 & 0.126 & -1.708 & 0.92 \\
$C e I I$ & $A_{\odot}(\mathrm{Ce})=1.70$ & \\
407.3374 & 0.478 & 0.230 & 1.52 \\
452.3075 & 0.516 & -0.030 & 1.54 \\
456.2359 & 0.478 & 0.230 & 1.54 \\
533.0556 & 0.869 & -0.400 & 1.68 \\
& \multicolumn{4}{ll}{} \\
$N d I I$ & $A_{\odot}(\mathrm{Nd})=1.45$ & \\
402.1327 & 0.321 & -0.100 & 1.35 \\
405.9950 & 0.205 & -0.520 & 1.59 \\
464.5760 & 0.559 & -0.760 & 1.50 \\
485.9026 & 0.321 & -0.440 & 1.59 \\
495.9115 & 0.064 & -0.800 & 1.62 \\
523.4190 & 0.550 & -0.510 & 1.56 \\
527.6869 & 0.859 & -0.440 & 1.30 \\
531.9810 & 0.550 & -0.140 & 1.52 \\
\hline
\end{tabular}

